\title{
Hardware Implementation of Communication Protocols modeled by Concurrent EFSMs with Multi-way Synchronization
}

\author{
Hisaaki Katagiri ${ }^{\dagger}$, Keiichi Yasumoto $\ddagger$, Akira Kitajima ${ }^{\dagger}$, \\ Teruo Higashino ${ }^{\dagger}$ and Kenichi Taniguchi \\ † Osaka Univ. \\ Graduate School of \\ Engineering Science \\ \{h-katagi,kitajima,higashino,taniguchi\}@ics.es.osaka-u.ac.jp \\ $\ddagger$ Shiga Univ. \\ Dept. of Information Processing \\ and Management \\ yasumoto@biwako.shiga-u.ac.jp
}

\begin{abstract}
In this paper, we propose a technique to implement communication protocols as hardware circuits using a model of concurrent EFSMs with multi-way synchronization. Since use of multi-way synchronization enables simple and comprehensible specifications of recent communication protocols which frequently use complicated mechanisms such as mutual exclusion and dynamic job assignment, the proposed model is expected to reduce development cost in designing/developing such protocols. We implement specifications described in the model so that EFSMs work synchronously with the same clock, and that the synchronization mechanism for checking executability of each tuple of synchronizing transitions is implemented as a combinational logic circuit. Through some experiments, we have confirmed that the proposed technique can synthesize hardware circuits with relatively good performances for practical use.
\end{abstract}

\section{General Terms}

high-level synthesis, concurrent EFSMs, multi-way synchronization, LOTOS, communication protocols

\section{INTRODUCTION}

For high-performance computer networking, hardware implementation of communication protocols has become important in recent years. In general, while developing high performance communication protocols, their internal algorithms and structures are usually modified repeatedly and simulations are applied until reaching expected performance. For reducing development cost, rapid prototyping techniques for automatic synthesis of hardware circuits from high-level specifications are essential in early stages of development.

For the purpose, researchers have begun focusing on the use of formal specification languages (formal description techniques: FDT) for communication protocols as starting points to synthesize hard- ware circuits for protocols [6, 9, 11, 14]. Using FDTs, message exchanges among concurrent processes such as multi-point/broadcast communication and mutual exclusion for accessing shared resources (e.g., FIFO queue) can be represented in a straightforward way. Since a lot of verification/validation techniques and the corresponding tools for FDTs are now available [2], hardware synthesis from protocol specifications in FDTs is useful to improve reliability of hardware circuits.

Several hardware implementation techniques for SDL and Estelle specifications have been proposed $[3,6,14]$. However, they require complicated operations for mutual exclusion in accessing shared resources among concurrent EFSMs since only one-to-one asynchronous communication between EFSMs is available in SDL and Estelle. On the other hand, LOTOS has a mechanism called multiway synchronization (which allows multiple concurrent processes to synchronize with each other by exchanging data when some conditions hold among the processes). It provides simplicity and comprehensibility in specifying complicated communication among multiple concurrent processes such as mutual exclusion and dynamic job assignment, which are frequently used in recent communication protocols. In addition, multi-way synchronization enables the constraint oriented specification style $[5,13]$ where each functional unit of a system is designed as an independent module and then appropriate constraints (described as processes) are specified among those modules so that they work cooperatively. This technique is useful, for example, when we frequently need to modify constraints among modules and/or modify internal structure of each functional unit.

In this paper, we propose a technique and a tool to convert protocol specifications modeled by concurrent EFSMs with multi-way synchronization into the corresponding RT-level VHDL descriptions. The corresponding hardware circuits can be synthesized using usual VHDL synthesis tools on the market. In our model, we suppose general EFSMs where each EFSM executes one of transitions outgoing from its current state. Each transition corresponds to an I/O action at a port (called gate in LOTOS literature) where multiple parameters can be transmitted in an I/O action. An execution condition (called guard) consisting of some variables and constants can be attached to each transition. In addition, any tuple of transitions with the same gate can be specified to synchronize with each other among a subset of EFSMs in order to exchange some values by multi-way synchronization. We implement specifications de- 
scribed in the proposed model so that EFSMs work synchronously with the same clock, and that the synchronization mechanism for checking executablity of each tuple of synchronizing transitions is implemented as a combinational logic circuit.

The proposed technique may take some overhead for implementing multi-way synchronization among EFSMs compared to other models without synchronization. Through some experiments in development of example circuits such as network switches and ATM switches, we have confirmed that hardware circuits synthesized with the proposed technique have good performances comparable to actual network products on the market. Since use of multiway synchronization provides simple and comprehensible design of communication protocols, the proposed technique will be useful to reduce development cost and improve reliability of circuits.

\subsection{Related work}

As related work, several high-level synthesis techniques which treat concurrent processes with their synchronization have been proposed $[1,4]$.

In [1], a technique to share resources among concurrent processes without conflicts based on the hierarchical scheduling technique is proposed. This technique first generates a communication dependency graph among processes, and then detects synchronization points for avoiding conflicts of shared resources using the graph. In this model, although designers do not have to specify mutual exclusion among processes, it may be costly to find synchronization points and scheduling of processes without conflicts.

In [4], an automatic conversion technique of system-level VHDL specifications into RT-level specifications is proposed. In this model, multi-point communication such as 1 to $\mathrm{N}$ and $\mathrm{M}$ to $\mathrm{N}$ is considered to improve simplicity/comprehensibility in system-level specifications. However, unlike multi-way synchronization of the proposed model, this model does not clearly handle more complicated multipoint communication such as dynamic selection among several exclusive synchronization tuples (i.e., mutual exclusion).

As LOTOS based synthesis techniques, in [10], a technique to convert timed LOTOS specifications to VHDL specifications has been proposed. However, only two-way synchronization between two processes is considered. [11] has also proposed a technique to synthesize hardware circuits from LOTOS specifications, but focuses only on Basic LOTOS [7] (i.e., LOTOS without data values).

Unlike the above techniques, in our model we specify communication protocols as concurrent EFSMs with multi-way synchronization where more complicated communication such as 1 to $\mathrm{N}$ data distribution and a random selection of a synchronization tuple among several exclusive ones can be easily specified.

\section{CONCURRENT EFSMS WITH MULTI- WAY SYNCHRONIZATION}

\section{1 multi-way synchronization}

In LOTOS [7], with the parallel operator with synchronization gates, we can specify multiple concurrent processes to execute some events and exchange data values in synchronization with each other (called multi-way synchronization).

$P[a, b, c]|[a, b]|(Q[a, b, d]|[a]| R[a, b])$

$(P[a, b, c]$ means $P$ uses three gates $a, b$ and $c$, and $\mid[$ gate_list $] \mid$
Table 1: Synchronization condition

\begin{tabular}{c|c|c|c}
\hline \hline$P_{i}$ & $P_{j}$ & condition & result \\
\hline \hline$g ! E_{i}$ & $g ! E_{j}$ & $\operatorname{val}\left(E_{i}\right)=\operatorname{val}\left(E_{j}\right)$ & - \\
$g ! E_{i}$ & $g ? x: t$ & $\operatorname{val}\left(E_{i}\right) \in \operatorname{domain}(t)$ & $x \leftarrow \operatorname{val}\left(E_{i}\right)$ \\
$g ? x: t$ & $g ? y: u$ & $t=u$ & $x, y \leftarrow$ Ext, \\
& & & Ext $\in \operatorname{domain}(t)$ \\
\hline
\end{tabular}

( $\operatorname{val}(E)$ is the value of $E, E x t$ is the value input from gate $g$ )

specifies multi-way synchronization among processes)

In the above example, processes $P, Q$ and $R$ must execute events on gate $a$ in synchronization with each other. On the other hand, events on gate $b$ can be executed between either processes $P$ and $Q$ or processes $P$ and $R$. Events on gates $c$ and $d$ can be executed in each process independently of the others. Value exchange among processes are also possible by multi-way synchronization. In each pair of synchronizing events, when one event is an output action (e.g., $a ! 3$ ) and the other is an input action (e.g., $a ? x:$ int $[p(x)]$ ), the output value is substituted to the variable $(x)$, if both the output value and the input variable have the same data type and guard expression $p(x)$ holds. When synchronizing events are all output actions, synchronization can be executed if all of the output values are the same. More formally, a tuple of synchronizing events can be executed by multi-way synchronization if and only if any pair of those events satisfies the conditions in Table 1.

\subsection{Definition of S-EFSMs model}

In S-EFSMs model, we suppose that each EFSM can have a finite number of registers (variables), that an execution condition called the guard expression can be specified to each transition, and that each transition can input/output several values via a gate as an $\mathrm{I} / \mathrm{O}$ action. Each transition is denoted by $g v[f]$ where $g$ is a gate, $v$ is a sequence consisting of input variables $(? x: t)$ and output expressions $(! E)$ on gate $g$, and $f$ is a guard expression of the transition.

In S-EFSMs model, any subset of concurrent EFSMs can communicate with each other via gates by multi-way synchronization. For the purpose, we borrow the parallel operators used in LOTOS. Finally, S-EFSMs are described by the following notation.

$$
S::=S \mid[\text { gate_list }]|S| S|| S \mid \text { EFSM }
$$

(EFSM denotes an EFSM. gate_list indicates the gates whose events have to be synchronized between its operands. ||| is an independent parallel operator, and it is defined as the special case of the parallel operator such that gate_list $=\emptyset$.)

\subsection{Extraction of rendezvous table}

Like LOTOS, multi-way synchronization in S-EFSMs is specified by just giving abstract relationships among concurrent EFSMs by the parallel operators. For the efficient implementation of multiway synchronization, we should calculate in advance the information about (i) the combinations of synchronizing EFSMs, (ii) the tuples of synchronizing transitions (synchronization tuples) and (iii) their execution conditions. If we count up all synchronization tuples among EFSMs, the number may be $O\left(k^{n}\right)$ in the worst case where $n$ and $k$ are the number of EFSMs and the number of transitions in an EFSM, respectively.

We represent all synchronization tuples by a set of rendezvous indications where each indication denotes a tuple of transition sets on a gate for a combination of synchronizing EFSMs. Here, every combination of transitions (i.e., every synchronization tuple) in the sets has the possibility to be executed by multi-way synchronization 


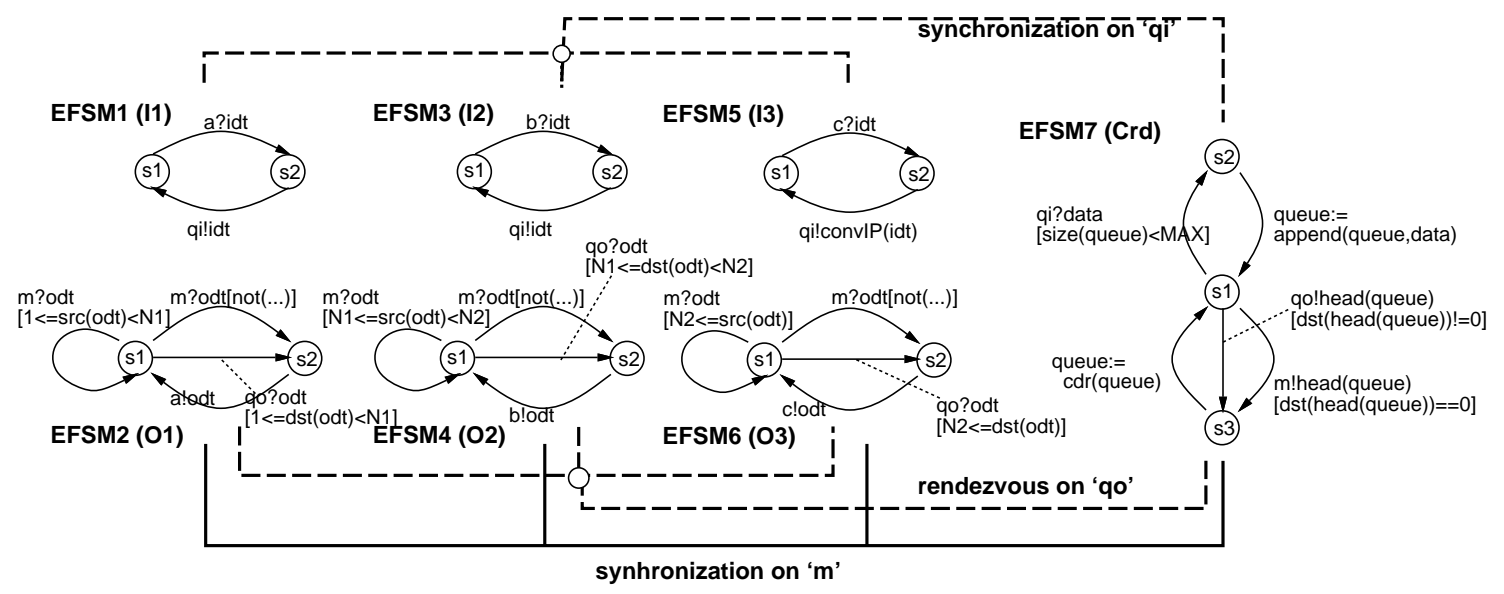

Figure 2: S-EFSMs for the network switch.

Table 2: The rendezvous table of the network switch

\begin{tabular}{|c|c|c|c|}
\hline No. & tuple of EFSMs & tuple of synchronization transition sets & confset \\
\hline$(1)$ & $(1,7)$ & $(\{(q i ! i d t$, true,-$)\},\{(q i ?$ data, size $(q u e u e)<M A X$, data $\leftarrow i d t)\})$ & $\{(2),(3),(4),(5),(6),(7)\}$ \\
\hline (2) & $(3,7)$ & $(\{($ qi!idt, true,-$)\},\{(q i ?$ data, size $($ queue $)<M A X$, data $\leftarrow i d t)\})$ & $\{(1),(3),(4),(5),(6),(7)\}$ \\
\hline (3) & $(5,7)$ & $(\{($ qi!idt, true,-$)\},\{(q i ?$ data, size $($ queue $)<M A X$, data $\leftarrow i d t)\})$ & $\{(1),(2),(4),(5),(6),(7)\}$ \\
\hline (4) & $(2,7)$ & $\begin{array}{l}\{(\text { qo?odt }, \text { dst }(\text { odt })<N 1, \text { odt } \leftarrow \text { head }(\text { queue }))\}, \\
\{(\text { qo!head }(\text { queue }), \text { dst }(\text { head }(\text { queue })) !=0,-)\})\end{array}$ & $\{(1),(2),(3),(5),(6),(7)\}$ \\
\hline (5) & $(4,7)$ & 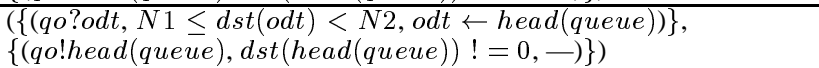 & $\{(1),(2),(3),(4),(6),(7)\}$ \\
\hline (6) & $(6,7)$ & $\begin{array}{l}(\{(\text { qo } ? \text { odt }, N 2 \leq \text { dst }(\text { odt }), \text { odt } \leftarrow \text { head }(\text { queue }))\}, \\
\{(\text { qo!head }(\text { queue }), \text { dst }(\text { head }(\text { queue })) !=0,-)\})\end{array}$ & $\{(1),(2),(3),(4),(5),(7)\}$ \\
\hline (7) & $(2,4,6,7)$ & 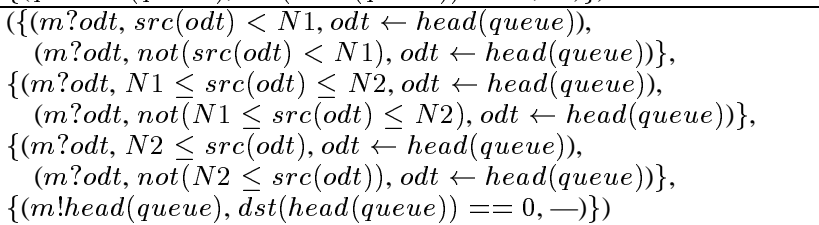 & $\{(1),(2),(3),(4),(5),(6)\}$ \\
\hline
\end{tabular}

holds. Therefore, the tuple (qi!idt, qi?data $[\operatorname{size}(q u e u e)<M A X])$ can be executed by the rendezvous indication (1) in Table 2. When the tuple is executed, the value of $i d t$ is assigned to the undefined variable data, and the current state is changed to $\left(s_{1}, s_{1}, s_{2}\right)$ in EFSM1, EFSM5 and EFSM7.

In some state, there may be several synchronization tuples to be executable simultaneously. For example, in Fig. 2, when the current state is $\left(s_{1}, s_{1}, s_{1}\right)$ for EFSM2, EFSM6 and EFSM7, a synchronization tuple (qo?odt [dst(odt) $<N 1$, qo!head(queue) $[\operatorname{dst}($ head $(q u e u e)) !=0]$ ) could be executed between EFSM2 and EFSM7 by the rendezvous indication (4) as well as (qo?odt $[N 2 \leq d s t($ odt $)]$, qo!head(queue) [dst(head(queue)) ! = 0]) between EFSM6 and EFSM7 by the rendezvous indication (6). In that case, one of them must be selected in a certain policy.

\section{HARDWARE CIRCUIT CONSTRUCTION}

In this section, we give the technique to convert given concurrent EFSMs with a rendezvous table into a synchronous sequential circuit. Hereafter, we suppose the modules corresponding to EFSMs work synchronously with the same clock. In each clock cycle, each EFSM can execute one transition as long as its execution condition holds. We assume that primitive hardware components such as arithmetic and boolean functions are provided as combinational logic circuits in advance and they can output the resulting values within one clock cycle. The circuit for each EFSM can be implemented easily as an FSM and datapath. So, here we concentrate on the implementation of multi-way synchronization mechanism among EFSMs.
Given EFSMs and a rendezvous table, we implement multi-way synchronization among EFSMs as the multi-way synchronization circuit consisting of the following three sub-parts: (1) executability check part checking whether there exist executable synchronization tuples for each rendezvous indication at each state; (2) data transfer part transferring the required data from a certain EFSM to the other EFSMs so that each EFSM can calculate the execution condition (guard) of its transition; (3) conflict avoidance part selecting a synchronization tuple among some mutually exclusive ones.

\section{Constructing executability checking and data transfer parts}

For the executability checking part, every EFSM $E_{i}$ in each rendezvous indication must check whether some transitions in its synchronization transition set are executable at the current state. So in each $E_{i}$, for every $r \in \mathcal{R}$, we provide a circuit generating an output signal $r_{i_{-}} o k$ which becomes true (i.e., 1) only when a transition in $A_{i}$ becomes executable. Consequently, for the rendezvous indication $r$ there exist some executable synchronization tuples if and only if all of $r_{1 \_} o k, \cdots, r_{m_{-}} o k$ (denoted by $r_{* \_} o k$ ) are true.

For the data transfer part, EFSMs with input transitions and an EFSM with output transitions can be determined statically for each rendezvous indication as described briefly in Sect. 2.3. Hence, we provide a path $D_{r}$ among EFSMs for each $r$ so that an EFSM outputs an appropriate value to the path and the others obtain the value.

\section{Constructing conflict avoidance part}


Table 3: An example of rendezvous table

\begin{tabular}{|c|c|c|c|}
\hline Info. \# & EFSMs & synchronization transition sets & confset \\
\hline$r_{1}$ & (EFSM1, EFSM2) & $\begin{array}{l}\left(\left\{\left(a ? x_{1}, p\left(x_{1}\right), x_{1} \leftarrow 1\right),\left(a ? x_{2}, q\left(x_{2}\right), x_{2} \leftarrow 1\right),\left(a ? x_{3}\right)\right\}\right. \\
\quad\{(a ! 1, \text { true },-)\})\end{array}$ & \\
\hline$r_{2}$ & (EFSM1, EFSM2) & $\begin{array}{l}\left(\left\{\left(a ? x_{1}, p\left(x_{1}\right), x_{1} \leftarrow g(y)\right),\left(a ? x_{2}, q\left(x_{2}\right), x_{2} \leftarrow g(y)\right),\left(a ? x_{3}\right)\right\}\right. \\
\quad\{(a ! g(y), \text { true },-)\})\end{array}$ & $\left\{r_{1}\right\}$ \\
\hline$r_{3}$ & (EFSM1, EFSM2) & $\left(\left\{\left(b ! f\left(x_{1}\right)\right.\right.\right.$, true,-$\left.)\right\},\left\{\left(b ? y\right.\right.$, true,$\left.\left.\left.y \leftarrow f\left(x_{1}\right)\right)\right\}\right)$ & $\emptyset$ \\
\hline
\end{tabular}

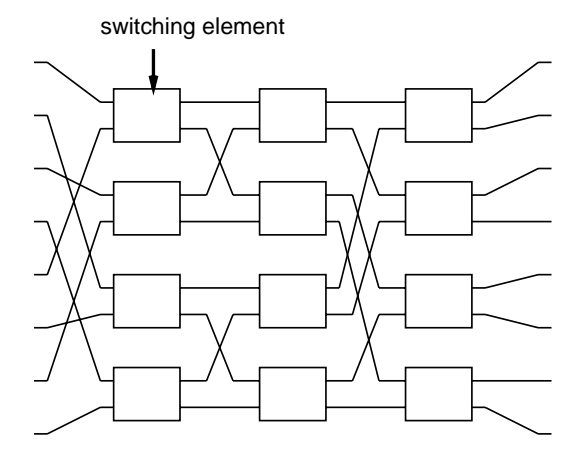

incoming cell outgoing cell

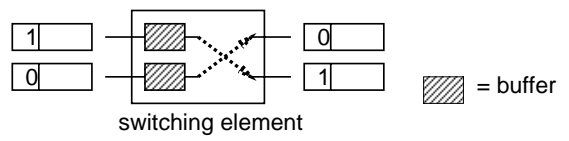

Figure 5: The Banyan switch.

Table 4: The number of logic gates and minimum clock period of various switching fabrics

\begin{tabular}{c|r|c}
\hline \hline switching fabric & \# of gates & min. clock period (ns) \\
\hline multi-bus fabric & 299,000 & 8.4 \\
banyan fabric & 202,000 & 7.4 \\
(batcher switch) & 401,000 & 6.6 \\
\hline
\end{tabular}

for example, in a buffer prepared at each input port. By placing a special device such as the Batcher switch [12] to re-order cells in front of the Banyan switch, cell conflict in switching elements can be avoided. The Batcher switch can also be composed of several switching elements with two lines.

In the experiment, we have described three types of switching fabric explained above: multi-bus; the Banyan switch; and the Batcher switch. The specifications could be described in S-EFSMs simply by describing each switching element as an EFSM and by specifying multi-way synchronization among those elements. The size and minimum clock cycles of the synthesized circuits are shown in Table 4 (here, we treated each cell as 53 byte parallel bits).

Usual bandwidth of a general ATM switch is about $150 \mathrm{Mbps}$. Thus, the maximum number of cell arrivals at each port is about 371,000 , and the time for processing each cell is about $2.8 \mu \mathrm{s}$. In $622 \mathrm{Mbps}$ ATM switches, the time will be about $700 \mathrm{~ns}$. From the result in Table 4, we can say that the circuits synthesized with our technique have good performances.

\section{CONCLUDED REMARKS}

In this paper, we have proposed a hardware implementation technique for communication protocols using a model of concurrent EFSMs with multi-way synchronization.

We have also developed tools to convert specifications in this model to the corresponding RT-level VHDL descriptions. We have confirmed that our technique and tools with usual synthesis tools on the market could synthesize hardware circuits with relatively good performances.

In the proposed technique, we suppose that each transition as well as each synchronization of transitions is executed in one clock cycle. To improve performance, it is adequate to implement each transition with relatively complicated functions as several transitions. Integrating such features to the proposed technique and treating timing constraints attached to events in E-LOTOS [8] are part of our future work.

\section{REFERENCES}

[1] O. Bringmann, W. Rosenstiel, and D. Reichardt. Synchronization detection for multi-process hierarchical synthesis. In International Symposium of System Synthesis (ISSS98), Dec. 1998.

[2] S. Chanson, H. Rudin, K. Turner, and S. Vuong. Formal description technique (FDT) concepts and tools. Computer Networks and ISDN Systems, 25(1), 1992.

[3] G. Csopaki and K. J. Turner. Modelling digital logic in SDL. In Proc. Joint Int. Conf. on Formal Description Techniques and Protocol Specification, Testing and Verification (FORTE/PSTV'97), pages 367-382, 1997.

[4] W. Ecker and M. Huber. VHDL-based communication- and synchronization synthesis. In European Design Automation Conference (EURO-DAC '95), Sept. 1995.

[5] M. Faci, L. Logrippo, and B. Stepien. Structural models for specifying telephone systems. Computer Networks and ISDN Systems, 29:501-528, 1997.

[6] W. Glunz and G. Venzl. Using SDL for hardware design. In Proc. 5th SDL Forum, 1991.

[7] ISO. Information Processing System, Open Systems Interconnection, LOTOS - A Formal Description Technique Based on the Temporal Ordering of Observational Behaviour, 1989. IS 8807.

[8] ISO/IEC JTC1/ SC21/ WG7. Final Committee Draft 15437 on Enhancements to LOTOS, 1998.

[9] C. D. Kloos and W. Damm, editors. Practical Formal Methods for Hardware Design, volume 1 of Research Reports Esprit, Project 6128, FORMAT. Springer, 1996.

[10] A. M. López, C. D. Kloos, T. R. Valladares, and T. M. Moro. Generating VHDL code from LOTOS Descriptions, pages 266-293. in [9], 1996.

[11] R. Sisto. A method to build symbolic representations of LOTOS specifications. In Proc. of 15th IFIP Int. Symp. on Protocol Specification, Testing and Verification (PSTV-XV), pages 331-346, 1995.

[12] A. S. Tanenbaum. Computer Networks. Prentice Hall, third edition, 1996.

[13] C. A. Vissers, G. Scollo, and M. v. Sinderen. Architecture and specification style in formal descriptions of distributed systems. In Proc. 8th Int. Symp. on Protocol Specification, Testing, and Verification (PSTV-VIII), pages 189-204, 1988.

[14] J. Wytrebowicz. Hardware specification generated from Estelle. In Proc. of 16th IFIP Int. Symp. on Protocol Specification, Testing and Verification (PSTV-XVI), pages 435-450, 1996.

[15] K. Yasumoto, A. Kitajima, T. Higashino, and K. Taniguchi. Hardware synthesis from protocol specifications in LOTOS. In Proc. 1998 IFIP Joint Intl. Conf. on Formal Description Techniques and Protocol Specification, Testing and Verification (FORTE/PSTV'98), pages 405-420, 1998. 